\title{
About the Profile Accuracy of the Involute Gear Hob
}

\author{
Márton MÁTÉ, Dénes HOLLANDA \\ Department of Mechanical Engineering, Faculty of Technical and Human Sciences, \\ Sapientia University, Tg. Mureş, \\ e-mail: mmate@ms.sapientia.ro,hollanda@ms.sapientia.ro
}

Manuscript received September 10, 2017; revised October 19, 2017.

\begin{abstract}
Gear hobs are the most widely and frequently used gear cutting tools. During the time passed between the moment of invention (Schiele, 1876) and the present, gear hobs reached a considerable evolution regarding the geometry, the profile of the edge, the relieving technologies finalizing in the latest constructive and design solutions. This paper deals with the calculus of the edge profile in the case the basic worm of the hob has involute helicoid surfaces. In order to obtain a constant grinding allowance on the relief faces of the gear hob teeth it is necessary to compute the edge of the roughing relieving cutter. The equations are deduced considering that the provenience involute worm is a one teethed helical gear with shifted profile. The presented mathematical model proves that linearizing the relieving cutter profile is not an adequate solution if aspiring to higher precision.
\end{abstract}

Keywords: gear hob, involute worm, edge, roughing, optimizing, profile

\section{Basic concepts regarding the precision of meshing with gear hobs}

Gear hobs are the most widely used cutting tools in the gear industry. It was invented in 1856 by the German Christian Schiele. The application of the invention followed later because that time the existing manufacturing technology was not able to ensure the constancy of the cutting edge profile after the re-sharpening operation. This handicap was eliminated by the invention of the relieving lathe, accorded to Friederich Mueller, an American engineer from Hartford, Connecticut (US Patent 1299207 A, 1916.)

The widely use of this excellent gear cutting tool started with the invention of the German engineer Herman Pfauter, who built up in his factory situated in Chemnitz the first universal gear hobbing machine.

The mathematical models of the gear hobs were widely studied that after. Technical literature contains an immense quantity of studies, papers, dissertations regarding different aspects of the geometry, profile precision and 
constructive solutions. Nowadays a large diversification of constructive solutions are on hands. This solutions offer large machining possibilities with increased cutting performance including the novel and very popular dry cutting technology.

Regarding the mathematical model of the cutting kinematics, two major geometric approaches can be defined. The first model considers that gear hob's generating surfaces are permanently tangent to the surfaces of a generating rack, and the rack is moving along its pitch line due to the helix effect that appears while the hob is rotating about its own axis $[1,2,5]$. Introducing the axial feed, the edges of the hob will sweep the all rack tooth surface. This model is almost everywhere accepted. As a consequence the meshing with a gear hob and with a planning comb is considered often to be equivalent. Litvin has demonstrated that this supposition can be accepted only as an approximation, because the helix effect introduces certain profile modifications [15].

The second geometric hypothesis, more appropriate in opinion of the authors, considers that gear hob's generating surface coincides with a one to utmost 5 teethed helical gear's tooth surface. This helical gear constitute with the machined gear a hyperbolic gear pair. In case of cutting of an involute gear, the generating surface most coinciding with an involute worm, meshed with a standard generating rack $[4,12,13,14]$.

As long as modern computing and simulation methods and environments were developed, research regarding the profile of cutting edge and cutting wedge surfaces marked a new evolution. Different mathematical models were developed regarding the meshing between a gear and an arbitrary rack [3, 8]. Nowadays sophisticated numerical control based manufacturing technologies allow the achieving of the most complex surfaces $[9,10]$.

However, standards indicate only the basic cutter profile of the gear hob [6] admitting that this is equivalent with the basic rack profile. Gear hob profile is considered to be the task of the manufacturers.

Regarding the peculiar aspects mentioned in the synthesis above the goal of present paper is to answer in which cases the linearization of the roughing relieving cutter edge or the grinding wheel profile is possible. In the followings the hypothesis of the involute basic worm of the gear hob is admitted.

\section{Theoretical and geometrical aspects of the basic worm's surface}

Based on the theoretical achievements presented above, this paper starts by admitting the second hypothesis described above related to gear hobbing. As a consequence, generating surface of the gear hob is an involute helicoid that is meshing with a standard rack, whose standard dimensions are defined in the normal section [11]. The pitch cylinder of the hob's basic involute worm is 
tangent to the pitch plane of the rack, while the axis of the worm is perpendicular to the direction of linear motion of the rack in case of reciprocate meshing. Here the main helix of the involute thread closes with the pitch line the angle $\lambda_{0}$, equal with the pitch helix angle of the involute thread [13, 14]. As a consequence the rack teeth declination angle becomes $\beta_{0}=\pi / 2-\lambda_{0}$. In case of one start thread the value of the declination angle must be smaller than $2^{\circ} 30$ ' that leads to a much brooded rack profile whose profile angle approaches almost $84^{\circ}$. The consequence is that the difference between the radiuses of the basic circle and the pitch circle are improper large. This fact involves improper involute curve segments as real generatrix-point manifolds for the involute helicoid surfaces as it is shown in Fig. 1.

It can be observed that the properties of a worm thread profile limiting involute arch are completely different from the involute arches that limits a classical spur gear tooth profile [16]. The profile shifting modifies the tooth thickness on the pitch circle. In case of one starting thread worm and zero profile shifting this is equal to the half of the pitch circle circumference, as it is stated by the position of the involute curves 1 and 1'. If a positive profile shifting exists the tooth thickness is increasing. Therefore pitch circle points of the tooth profiles present not anymore diametric symmetry as it can be observed on curves 2 and 2'. Due to the particularly involute curve segments situated between the pitch and the addendum circle, in contrarious with the case of involute tooth, the topland width increases while increasing the profile shifting parameter. Due to this fact, increasing the profile shifting leads to a massive hob tooth that is disadvantageous as it generates large dedendum transition curves on the meshed gear tooth profile. From this reasons the basic worm of the gear hob is accepted as a helical involute one teethed Willis type gear.

The classical parametric equations of the involute curve $[11,15]$ are not advantageous for computing the useful subset of the involute helix surface due to the fact that the rolling parameter of the involute generating line - the $\varphi$ angle - get there values starting from zero, and the useful subset mentioned before needs values grater then $\pi$ radians more difficult to be perceptible. According to this, involute arch $l$ is drawn by the extremity $A_{l}$ of the segment $A A_{l}$, while the half-line opposite to $A A_{l}$ rolls on the basic circle.

For an arbitrary position set by the value $\varphi$ of the rolling parameter, the segment $A A_{l}$ becomes $B B_{l}$ and point where $B$ ' matches the following coordinates:

$$
\left\{\begin{array}{l}
x(\varphi)=R_{b}\left(\cos E(\varphi)+\left(\tan \alpha_{0 t}+\varphi\right) \sin E(\varphi)\right) \\
y(\varphi)=j R_{b}\left(\sin E(\varphi)-\left(\tan \alpha_{0 t}+\varphi\right) \cos E(\varphi)\right)
\end{array}\right.
$$




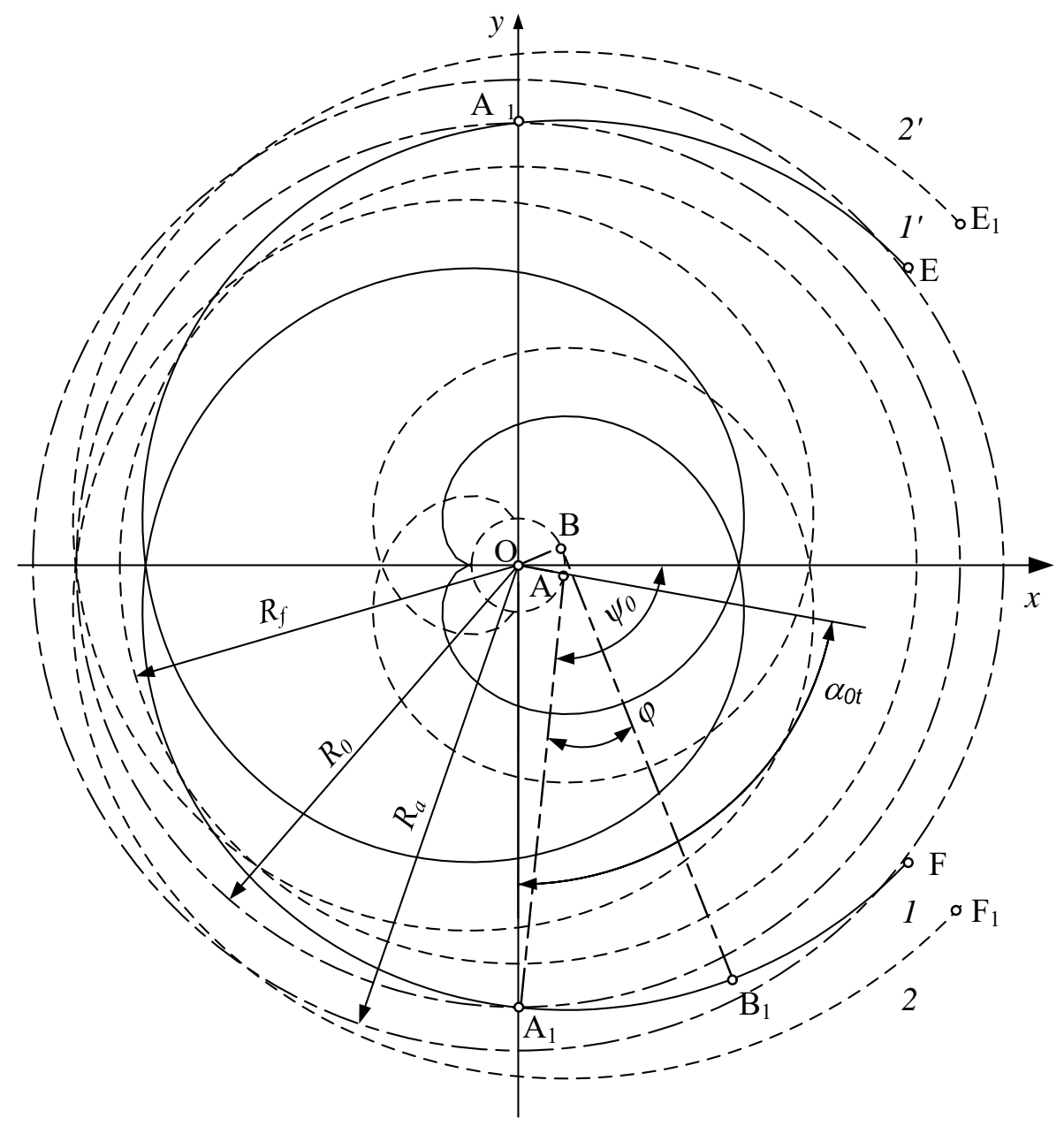

Figure 1: The complete generating curve of an involute worm (radial section)

Here the argument of sine and cosine function has the form

$$
\left\{\begin{array}{l}
E(\varphi)=\varphi-\psi_{0}\left(\Delta_{x}\right)+\alpha_{0 t}, \\
\psi_{0}\left(\Delta_{x}\right)=\frac{\pi}{2}+2 \frac{\Delta_{x}}{m_{t}} \tan \alpha_{0 t}
\end{array}\right.
$$

where $\Delta_{x}$ denotes the profile shifting. 
Parameter $j$ differentiates the involute arches who define the cross section of the worm. If $j=1$ equations (1) refer the arch 1 , for $j=-1$ they describe the opposite arch 1 '.

Using the second fundamental equation of the involute trigonometry [16] or the parametrical polar form of the involute,

$$
\left\{\begin{array}{l}
\rho(\varphi)=R_{b} \sqrt{1+\left(\tan \alpha_{0 t}+\varphi\right)^{2}} \\
\theta(\varphi)=\left(\tan \alpha_{0 t}+\varphi\right)-\arctan \left(\tan \alpha_{0 t}+\varphi\right)-\psi_{0}\left(\Delta_{x}\right)
\end{array}\right.
$$

it can be proven that topland width increases with the profile shifting.

The equations of the involute worm surfaces are obtained through a rototranslation of the involute profile along the axis $z$. Excepting the transformation matrix [11] and the elementary calculus the equations result in the following form:

$$
\left\{\begin{aligned}
& x(\varphi, u)=R_{b}\left(\cos E_{1}(\varphi, u)+\left(\tan \alpha_{0 t}+\varphi\right) \sin E_{1}(\varphi, u)\right) \\
& y(\varphi, u)=j R_{b}\left(\sin E_{1}(\varphi, u)+\left(\tan \alpha_{0 t}+\varphi\right) \cos E_{1}(\varphi, u)\right) \\
& z(\varphi, u)=\frac{p_{a x}}{2 \pi} u=\frac{m_{n}}{2 \cos \lambda_{0}} u \\
& E_{1}(\varphi, u)=E(\varphi)+j u, j \in\{-1 ; 1\}
\end{aligned}\right.
$$

It is easy to observe that the structure of equations (4) is similar to equations (2) of the involute. However involute worm gear's equations can be re-written using the equations of the generating line that rolls on the basic helix [7], but in the following calculuses with this would lead to more complicated equations.

\section{The gear hob derived from an involute worm}

As defined in the literature gear hob is a worm that is endowed with cutting properties. Therefore cutting edges and cutting wedge surfaces must be created. The simple intersection of the worm surface with another helicoid is not enough while relief surfaces giving positive relief angles will not be produced. For achieve this, relieving operation must be applied. Nowadays relieving is realized on relieving lathes, both the roughing by cutting and the final grinding. The tooth resulted after the relieving operation present helicoid surfaces based on a conical helix directory. Practically, re-sharpening ensures the edge form constancy $[7,12]$. Theoretically it was proven that the edge form of the spiroid gear hobs variates with the re-sharpening [11]. This effect appear at the 
cylindrical gear hob edges too, but it is neglectable since the modification is lower than $10^{-4} \mathrm{~mm}$. The problem appears due to the fact that the re-sharpened edge cannot rebuild the original involute worm in the gearing process. Resharpening operations lead to diameter decreasing, helix angle increasing and as a consequence - to the modification of the curvature of helical surfaces of the equivalent worm. The equivalent worm can be defined as a worm whose helical surfaces include the edges of the re-sharpened hob. Errors of the involute profile obtained by hobbing, in case of admitting the perfect involute helical worm, are calculated in [15].

The main question that can be put regarding the theoretical and the geometrical peculiarities described before is how the best approximation of the involute worm can be obtained. The answer is given here by the model of the cutting edges. First cutting edges are obtained if intersecting the surfaces of the worm given by equations (4) with a helical rake face whose main helix line is perpendicular to the main helix line of the worm. Thus, the pitch $p_{C}$ of the rake face results from the condition of perpendicularity applied on the outstretched pitch cylinder [7]:

$$
\tan \lambda_{0}=\frac{p_{a x}}{2 \pi R_{0}}=\frac{2 \pi R_{0}}{p_{C}}
$$

The rake face of the hob is a linear helicoid whose generatrix is a straight line intersecting the axis and perpendicular to this. The directory of the surface is a cylindrical helix that fits the pitch cylinder and perpendicular to the pitch helix. Mathematically it can be described by the rototranslation of a line coincident with axis $x$ using a helical direction opposite to the worm. After elementary calculus the equations of the rake face are the followings:

$$
\left\{\begin{array}{l}
x(t, v)=t \cos v \\
y(t, v)=t \sin v \\
z(t, v)=-\frac{p_{C}}{2 \pi} v
\end{array}\right.
$$

Fig. 2 shows the helix surfaces of the involute worm intersected by the helical rake face.

The equations of the theoretical cutting edges result from equations (4) and (7). Here a dependence between the independent parameters $\varphi, u$ of the helix surfaces $\Sigma_{l}$ and $\Sigma_{r}$ must be obtained. The easiest way is to transform the rake face's equations in an implicit form by eliminating parameters $u$ and $t$ : 


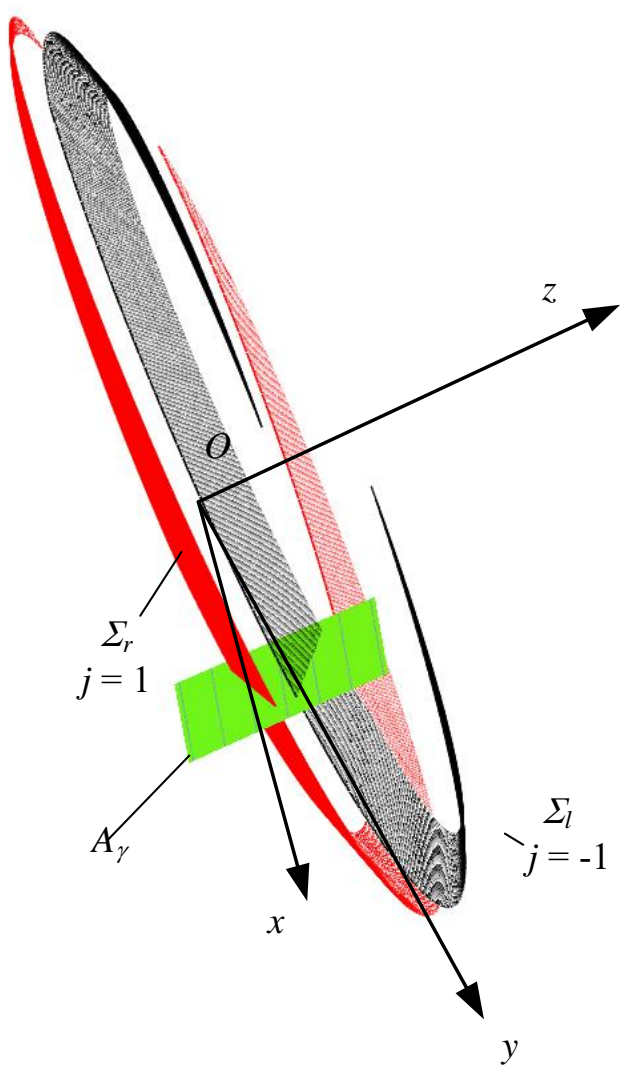

Figure 2: The involute worm thread limiting surfaces and the rake face

$$
z+\frac{p_{C}}{2 \pi} \arctan \frac{y}{x}=0
$$

Now the coordinate functions (4) are implemented in equation (7) that becomes of form $\Phi(\varphi, u)=0$. In order to simplify the expression of the solution, some variable changings are necessary. Thus,

$$
\begin{aligned}
& E_{1}(\varphi, u)=\varphi-\psi_{0}+\alpha_{0 t}+j u=-\psi_{0}+\left(\varphi+\tan \alpha_{0 t}\right)-\tan \alpha_{0 t}+\alpha_{0 t}+j u= \\
& =-\left(\psi_{0}+\operatorname{inv} \alpha_{0 t}\right)+\varphi_{1}+j u=-\zeta+\varphi_{1}+j u
\end{aligned}
$$

and using this form the solution of $\Phi(\varphi, u)=0$ regarding to parameter $u$ is the following:

$$
u\left(\varphi_{1} ; j\right)=j \frac{p_{C} p_{a x}}{p_{C}+p_{a x}}\left(\zeta+\arctan \varphi_{1}-\varphi_{1}\right)
$$


Finally, replacing the parameter $u$ in (4) by the function (9) and using the transformations (8), the unified equations for both edges result as:

$$
\left\{\begin{array}{l}
x\left(\varphi_{1} ; j\right)=R_{b}\left(\cos B\left(\varphi_{1}\right)+\varphi_{1} \sin B\left(\varphi_{1}\right)\right) \\
y\left(\varphi_{1} ; j\right)=j R_{b}\left(\sin B\left(\varphi_{1}\right)-\varphi_{1} \cos B\left(\varphi_{1}\right)\right) \\
z\left(\varphi_{1} ; j\right)=j \frac{p_{C} p_{a x}}{p_{C}+p_{a x}}\left(\zeta+\arctan \varphi_{1}-\varphi_{1}\right) \\
B\left(\varphi_{1}\right)=\frac{p_{a x}}{p_{C}+p_{a x}}\left(\varphi_{1}-\zeta\right)+\frac{p_{C}}{p_{C}+p_{a x}} \arctan \varphi_{1}
\end{array}\right.
$$

The edges and the rake face are shown in Fig. 3.

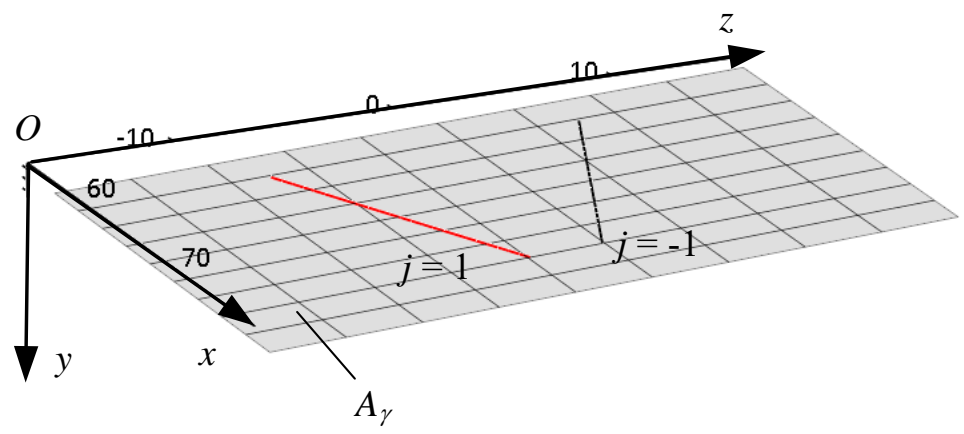

Figure 3: The theoretical cutting edges and the rake face

\section{Manufacturing peculiarities}

As well as known, relief faces of the gear-hob teeth are obtained by relieving turning and grinding, on the relieving lathe $[1,2,5,7,17]$. In order to reduce the manufacturing costs the investments in relieving operations are to be minimized. A fair solution can be obtained if the relief faces are linearized e.g. the generatrix of the surfaces must be a straight line. This leads to the aim of linearizing the cutting edges, while in this case the costs with the relieving cutter and the grinding wheel are considerably reduced. Even in case the precision of the edges are not acceptable still result a rough surface that presents a quasi-equal grinding allowance repartition that is advantageous. As a conclusion, the linearization of the cutting edges produce lowered 
manufacturing costs or a higher precision of the grinding realized with a wheel dressed about a curved profile.

Let's suppose that the cutting edge results as intersection of two perpendicular planes

$$
x+\alpha_{i} y+\beta_{i} y+\gamma_{i}=0, i \in\{1 ; 2\}, \alpha_{i}, \beta_{i}, \gamma_{i} \in \mathfrak{R}
$$

Now let's consider $N$ equidistant points along the edge chosen as subject of linearization. The more the edgepoint is distanced from the line, the absolute value of the right side of equations (11) raises. As a consequence, the objective function can be defined as the sum of squares of left sides:

$$
\mathrm{F}\left(\alpha_{i}, \beta_{i,}, \gamma_{i}\right)=\sum_{i=1}^{2} \sum_{j=1}^{N}\left(x_{j}+\alpha_{i} y_{j}+\beta_{i} z_{j}+\gamma_{i}\right)^{2} \rightarrow \min
$$

The objective function written in this form cannot be used, due to the symmetry related to index $i$. This causes the rank of the linear system of six equations built up of the partial derivatives of $\mathrm{F}$ is only 3 while the number of unknowns, $\alpha_{i}, \beta_{i}, \gamma_{i}, i \in\{1 ; 2\}$ is 6 . The given situation can be overstepped by applying the Lagrangian multipliers method. Accepting the approximating line resulting as intersection of perpendicular planes, it is obvious that

$$
1+\alpha_{1} \alpha_{2}+\beta_{1} \beta_{2}=0
$$

Using the constraint (13) whose subject is function (12) the new objective function results as

$$
\mathrm{F}_{1}\left(\alpha_{i}, \beta_{i}, \gamma_{i}, \lambda\right)=\mathrm{F}\left(\alpha_{i}, \beta_{i}, \gamma_{i}\right)+\lambda\left(1+\alpha_{1} \alpha_{2}+\beta_{1} \beta_{2}\right) \rightarrow \min
$$

The system built up of the partial derivatives of the objective function in 7 unknowns is the following:

$$
\left\{\begin{array}{l}
\frac{\partial \mathrm{F}_{1}}{\partial q}=0, q \in\left\{\alpha_{i}, \beta_{i}, \gamma_{i}, \lambda\right\}, i \in\{1 ; 2\} \\
\frac{\partial \mathrm{F}_{1}}{\partial \lambda}=0
\end{array}\right.
$$

This is a nonlinear system that is recommended to be handled numerically.

In order to minimize the number of iterations the initial position of the intersected planes is chosen in the vicinity of the edgepoint situated on the pitch cylinder. Let's denote $R_{0}$ the radius of the pitch cylinder. Using the $x$ and $y$ coordinate functions (10), the value of the parameter $\varphi_{1}$ results as 


$$
\varphi^{\left({ }^{(0)}\right.}=\sqrt{\frac{R_{0}^{2}}{R_{b}^{2}}-1}
$$

A good first approximation for the normal vectors of the planes can be deduced involving the geometrical elements presented in Fig. 4. Let's denote $A$ the intersection point of the pitch cylinder generatrix $\kappa_{0}-\kappa_{0}$ with that tangent line of the rake face's pitch helix $\tau_{0}-\tau_{0}$ that intersects the axis $x$.

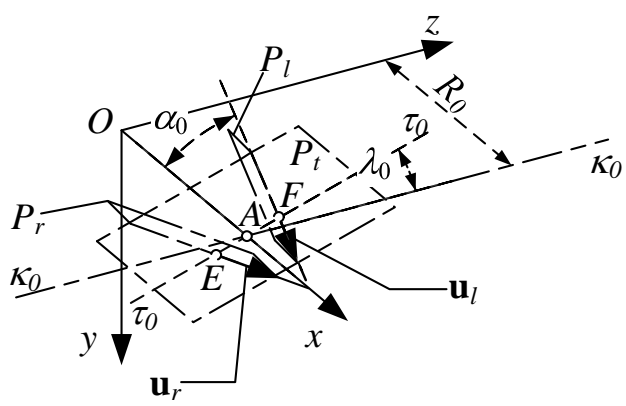

Figure 4: The geometrical elements involved in the computing of the first solution

The plane $P_{t}$ is built on the axis $x$ and the pitch helix tangent. Planes $P_{r}$ respectively $P_{l}$ are perpendicular to $P_{t}$ intersecting this along the profile lines of the normal rake, declined by $\alpha_{0}$ to the axis $x$. Thus $A E=A F=\frac{\pi m_{n}}{4}=a$, the coordinates of points $E$ and $F$ with respect of the values -1 and 1 of the switch parameter $j$ are

$$
\underline{\mathbf{r}}=\left(\begin{array}{lll}
R_{0} & -j a \cos \lambda_{0} & -j a \sin \lambda_{0}
\end{array}\right)^{T}
$$

In the same way the direction unit vectors of the profile lines can be written as

$$
\underline{\mathbf{u}}_{r, l}=\left(\begin{array}{lll}
\cos \alpha_{0} & -j \sin \lambda_{0} \sin \alpha_{0} & j \cos \lambda_{0} \sin \alpha_{0}
\end{array}\right)^{T}
$$

Using the normal unit vector of the plane $P_{t}$ of coordinates

$$
\underline{\mathbf{n}}_{t}=\left(\begin{array}{lll}
0 & -\cos \lambda_{0} & -\sin \lambda_{0}
\end{array}\right)^{T}
$$

and expression (18), the normal vectors of the planes $P_{r}$ respectively $P_{l}$ can be computed as the cross product $\mathbf{n}_{t} \times \mathbf{u}_{r, l}$. Using the coordinates (17) of the points $E$ 
respectively $F$ the equations of the planes mentioned before can be written. Applying elementary transformations to these the first approximation of values $\alpha_{i}, \beta_{i}, \gamma_{i}, i=\overline{1,2}$ will be obtained.

\section{The distribution of the distances from the theoretical edge to the approximant optimum.}

Let's denote the solution of the system (16) built up for the left or the right edge with $\left(\alpha_{1}^{0}, \beta_{1}^{0}, \gamma_{1}^{0}, \alpha_{2}^{0}, \beta_{2}^{0}, \gamma_{2}^{0}\right)$. The value of $\lambda$ is not affecting the position of the best approximant. Now solving the linear system (11) with coefficients replaced by the correspondent values given through the solution of the system (16), the coordinates of the approximant line's characteristic point $M$ result as follows:

$$
\begin{aligned}
& x^{(M)}=\frac{\alpha_{1}^{0}\left(\beta_{2}^{0} t+\gamma_{2}^{0}\right)-\alpha_{2}^{0}\left(\beta_{1}^{0} t+\gamma_{1}^{0}\right)}{\alpha_{2}^{0}-\alpha_{1}^{0}} \\
& y^{(M)}=\frac{\left(\beta_{1}^{0} t+\gamma_{1}^{0}\right)-\left(\beta_{2}^{0} t+\gamma_{2}^{0}\right)}{\alpha_{2}^{0}-\alpha_{1}^{0}} \\
& z^{(M)}=t
\end{aligned}
$$

Here, for the simplifying of the formulae let's accept $t=0$.

The error is defined as the distance from the theoretical edgepoint to the approximant line. Recognizing here the classical analytical geometry problem of the distance from a given point to a line it can be written the distance as the module of the cross product computed with the unit vector $\mathbf{e}$ of the line and the vector binding the external point $A$ of the theoretical edge with an arbitrary point of the line, in this case $M$. Thus, it can be written the distance as

$$
d=|\mathbf{A M} \times \mathbf{e}|
$$

where the unit vector's coordinates are

$$
\underline{\mathbf{e}}=\frac{1}{\sqrt{\left(\alpha_{1}^{0} \beta_{2}^{0}-\beta_{1}^{0} \alpha_{2}^{0}\right)^{2}+\left(\beta_{1}^{0}-\beta_{2}^{0}\right)^{2}+\left(\alpha_{1}^{0}-\alpha_{2}^{0}\right)^{2}}}\left(\begin{array}{c}
\alpha_{1}^{0} \beta_{2}^{0}-\beta_{1}^{0} \alpha_{2}^{0} \\
\beta_{1}^{0}-\beta_{2}^{0} \\
-\alpha_{1}^{0}+\alpha_{2}^{0}
\end{array}\right)
$$




\section{Numerical results}

The mathematical model described above was tested for a gear hob derived from a basic involute worm of one tooth, for a normal module value $m_{n}=5 \mathrm{~mm}$ and a normal rack profile of $\alpha_{0}=20^{\circ}$. Eight values of the pitch helix angle were considered in arithmetic progression, starting from $\lambda_{0}=2^{\circ}$ till $\lambda_{0}=3^{\circ} 45^{\prime}$ with an increment of $\Delta_{\lambda 0}=15^{\prime}$. The computed distributions of the errors are presented in Fig. 5.

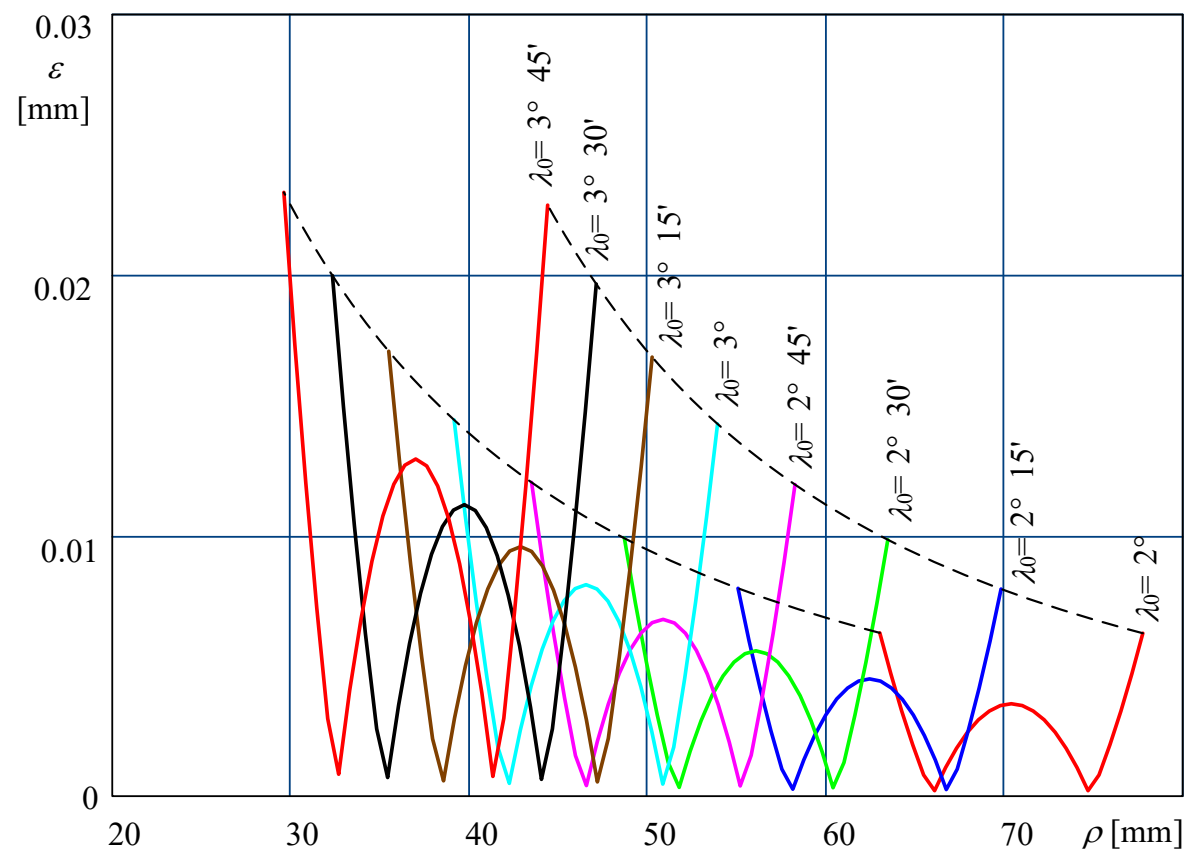

Figure 5: The geometrical elements involved in the computing of the first solution

Studying the shape of the error distribution curves the following remarks can be made:

- the best approximation lines doesn't intersect the theoretical edge;

- the error curves present a local maximum in the vicinity of the theoretical edgepoint situated on the pitch cylinder;

- the error's absolute maximum values are approximately equal and they are situated near the extremities of the theoretical edge;

- the maximum value of the error increases exponential with the pitch helix angle. 
If computing the maximum error value for the intermediate value of the helix angle $\lambda_{0}=2^{\circ} 45^{\prime}$ and for the module values of $1.25,2.5,5,8$ and $10 \mathrm{~mm}$ (according to DIN780) it will proved that the error is in linear dependence with the module.

The results above confirm the maximum error approximating formula must be of following form:

$$
\varepsilon=C \lambda_{0}^{x} m^{y}
$$

Using the data deduced on the parameter values described before, formula (23) becomes

$$
\varepsilon=1,324841 \lambda_{0}^{2,077046} m
$$

with a level of Pearson's $r$-correlation of $0,999990362592$.

\section{Conclusion}

As the mathematical model confirms, the pitch helix angle has a strong influence on the edge profile errors when the linearization is attempted.

The small values of the helix angles, e.g. $\lambda_{0}<2^{\circ}$ the errors are acceptable, but the pitch diameter of the hob strongly increases that leads to exaggerated material consumption, especially in case of large modules.

By helix angles larger than $2^{\circ}$, for finishing precision the linearization of the edges, the relieving using straight line profiled grinding wheels is not admitted. Here curved tooth profiles must be machined.

The linearized edges lead to irregular convolute worms, because they are not crossing lines.

The empirical formula can be used for computing the probable value of the maximal error while the edges are linearized. If the admissible value of error is given, formula (23) allows the computing of the maximum value of the pitch helix angle. Using this value, the frontal module and the pitch diameter of the theoretical involute worm can be computed. This led to the smallest possible diameters in case of linearized edges. If the addendum diameter exceeds the maximum admissible value, the decreasing is possible only if curved edges are applied. 


\section{References}

[1] Liston, K., “Hob Basics. Part I.”, in Gear technology magazine, vol. 10, nr.5, 1993, pp. 4652.

[2] Liston, K., "Hob Basics. Part II.”, in Gear technology magazine, vol. 10, nr.6, 1993, pp. 18 24.

[3] Sandeep M., Vijayakar, A. e.a., "Gear Tooth Profile Determination from Arbitrary Rack Geometry", in Gear technology magazine, vol. 5, nr. 6, 1988, pp. 18-30.

[4] Innocenti, C., (2007). "Optimal Choice of a Shaft Angle for Involute Gear Hobbing”, in Gear technology magazine, vol. 24, nr. 8, 2007, pp. 42-50.

[5] Yefim, Kotlyar, (2000). "Precision Finish Hobbing", in Gear technology magazine, vol. 17, $n r .4,2000$. pp. 22-26. Retrieved on 16.04.2015, from http://www.lmt-tools.de/ verzkat/page119.html \#/124

[6] *** DIN 3972. Wälzfräser-Bezugsprofile

[7] Hollanda, D., "Bazele așchierii și generării suprafețelor II". lecture notes, Petru Maior University of Tîrgu-Mureș, 1994.

[8] Dudás, I., Bányai, K., Varga, Gy., "Simulation of meshing of worm gearing", in American Society of Mechanical Engineers, Design Engineering Division (Publication) DE 88, 1996, pp. 141-146.

[9] Dudás, I., Varga, Gy., Bányai, K., "Holonic manufacturing system for production of different sophisticated surfaces", in Proceedings of the IASTED International Conference on Modelling, Simulation, and Optimization, 2004, pp. 72-75.

[10] Varga, Gy., Balajti, Zs., Dudás, I., "Advantages of the CCD camera measurements for profile and wear of cutting tools", in Journal of Physics: Conference Series 13 (1), 2005, pp. 159-162.

[11] Dudás, I., „The Theory and Practice of Worm Gear Drives”, Penton Press, 2005.

[12] Gyenge, Cs., "Determinarea profilului sculelor abrazive la detalonarea radială a frezelormelc" in Tehnologii Calitate Mașini Materiale Vol.7. București: Editura Tehnică, 1990, pp. 92-115.

[13] Gyenge, Cs., "Nagy pontosságú csigakerék-lefejtőmarók tervezése és gyártása” in Gép 43:(11-12), pp. 385-394.

[14] Gyenge, Cs., “A Frenet-féle triéder alkalmazása a csavarfelületek gyártástervezésében”, in Gépgyártástechnológia 32:(5-6), 1992, pp. 191-194.

[15] Litvin, F.L., Fuentes, A., "Gear Geometry and Applied Theory", Cambridge University Press.

[16] Szeniczei, L., “Általános fogazás”, Müszaki Nehézipari Könyvkiadó, Budapest,1958.

[17] Radzewich, S. P., "Gear Cutting Tools", CRC Press, NY, 2010. 